\title{
Radiology in the Undergraduate Medical Curriculum: Too Little, Too Late?
}

\author{
Adam A. Dmytriw ${ }^{1}$ - Philip S. Mok ${ }^{1}$ Natalia Gorelik ${ }^{3}$ - Jordan Kavanaugh ${ }^{2}$. \\ Peter Brown $^{2}$
}

Published online: 18 April 2015

(C) International Association of Medical Science Educators 2015

\begin{abstract}
There is an anecdotal need to increase radiology education in medical school. Surveys were distributed to three medical schools, with a respondency of $55 \%$. Over $91 \%$ of students believed there should be more radiology teaching in medical school. Students prefer different methods of teaching, lectures, group learning, and web-based modules.
\end{abstract}

Keywords Medical education · Curriculum · Radiology · Anatomy · Survey

\section{Introduction}

Over the past decade, there has been mounting evidence that medical students are not receiving adequate education in radiology [1]. In a time when medical curricula, including ours, are seeing significant modernization, the need for satisfactory comprehension of radiology by medical students has never been more pressing. As explicit teaching in anatomy declines, it increasingly falls to departments and instructors in radiology to ensure the adequacy of radiology teaching as a supplement to anatomy teaching [2]. Current studies suggest that as few as $25 \%$ of medical school graduates are confident in their appraisal of chest $\mathrm{x}$-rays [3]. Upon increasing the amount of

Adam A. Dmytriw

adam.dmytriw@uhn.ca

1 Department of Medical Imaging, University of Toronto, 263 McCaul St, Toronto, ON M5T 1W7, Canada

2 Department of Diagnostic Radiology, Dalhousie Medical School, 5850 College Street, Halifax, NS B3H 1X5, Canada

3 Department of Diagnostic Radiology, Faculty of Medicine, McGill University, 1650 Cedar Avenue, Montreal, QC H3G 1A4, Canada radiology teaching in medical school, students have been shown both to demonstrate more preparedness in clerkship and beyond, as well as an increased level of confidence. Moreover, instruction in radiology has been shown to improve confidence in physical examination [4]. Though the majority of medical students in a given class are not interested in specializing in radiology, it is clear that education in radiology is necessary for independent medical image interpretation and perhaps more importantly test workup and requisition [5]. Ultimately, this has led to an increased demand in the student population for instruction in radiology [6] and explicitly radiologist instructors [7].

At our institutions, the amount of radiology teaching in the undergraduate medical school curriculum given by radiologists has decreased over the past several years. As a result, medical student interest groups have asked for extracurricular radiology lectures. The purpose of our study was to formally determine if there was a perceived need for increased radiology teaching and exposure in the Dalhousie University, McGill University, and University of Toronto undergraduate medical curricula.

\section{Methods}

We conducted a multi-institutional survey asking all medical students to provide their impression of radiology education in the current undergraduate medical school curriculum.

Questions asked included those on the importance of radiology education, whether or not the amount of radiology teaching is adequate and how confident students feel in their ability to interpret basic x-rays. Responses were gauged on a Likert-type scale (e.g., critically important, very important, somewhat important, slightly important, or not at all important). In addition, students were also asked what radiology 
teaching methods they would like to see incorporated into future iterations of the curriculum. Surveys were distributed to the classes either on paper or online through Opinio, as students were not necessarily taking rotations at a local site. Results were tabulated after all surveys had been collected, both as all-student totals as well as a pre-clerkship (first and second year) and clerkship totals (third and fourth year).

\section{Results}

Overall response rate was $55 \%(1223 / 2224)$. Seventy-two percent of first-year, $74 \%$ of second-year, $32 \%$ of all thirdyear, and $59 \%$ of fourth-year students responded. When asked how important the radiologist was to the care team, over $91 \%$ of respondents believed radiologists were "critically important" or "very important" to the healthcare team (Fig. 1).

Ninety-eight percent of students reported that a basic understanding of radiology concepts was important to their future medical practice, with $34 \%$ indicating "critically important", $55 \%$ indicating "very important". Eighty-three percent felt that the amount of radiology education in the medical school curriculum was either "inadequate" (59\% of pre-clerkship, $70 \%$ of clerkship) or "very inadequate" (16\% of pre-clerkship, $22 \%$ of clerkship).

When asked about the amount of radiology teaching in the medical school curriculum, $63 \%$ of students believed it was "inadequate" ( $59 \%$ of pre-clerkship, $70 \%$ of clerkship), and $19 \%$ of students responded with "very inadequate" (16\% of pre-clerkship, $22 \%$ of clerkship) (Fig. 2).
Fig. 1 Survey distributed to medical students

\section{Radlology Education Survey For Medical Students}

1. What year of medical school training are you currently in?

Q Med 1

O Med 2

- Med 3

Med 4

2. In your opinion, how Important is the radlologist as a member of the heaith care team?
O Critically Important
- Very Importan:
o somewhat important
O slightly important
O Not at al Important

3. How Important is a basic understanding of general radiology concepts to your future medical practice? O Critically Important

- Very important

O Someshat important

O slightly important

Not at al important

4. The amount of radiology teachingreducation in the current medical school curriculum is:

O Excessive

- More than adequate

O Adequate

o Inadequate

- Very Inadequate

5. At the end of your medical school training, how confident wil you feel in your abllity to adequately Interpret the findings on baslc radlology studles, such as chest $x$-rays, abdominal $x$-rays, and bone $x$-rays.

o completely confident

Q Very confident

- Someshat confident

- silghtly conndent

O Not at al confident

6. In terms of the amount of radiology educationiteaching in the curriculum, which of the following statements do you agree with?

O Much more teaching is needed

- A little more teaching is needed

- The current amount of teaching is enough. No more is needed.

o Less radlology teaching should be done.

7. If you do want more radiology teaching incorporated into the curriculum, in what way should this be done? Please rank the options below.

Didactic lectures

Group leaming sesslons

Web based leaming modules

- Tutorial based cases

- Other (olease specify) 

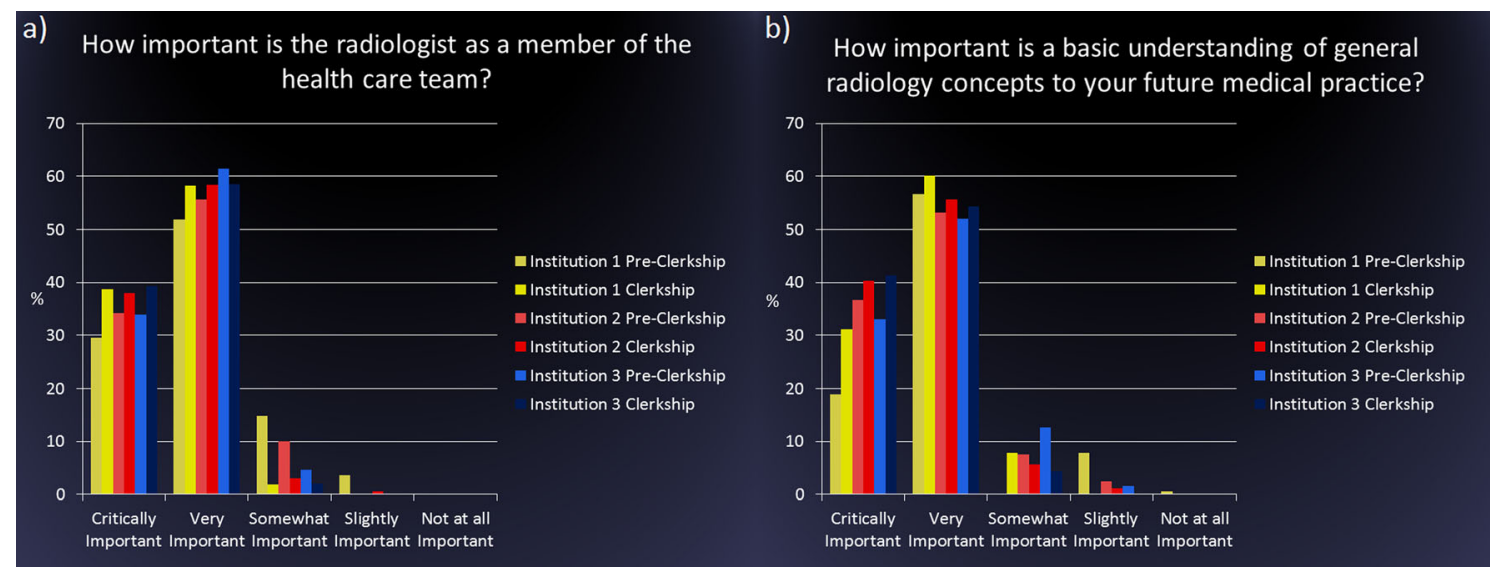

Fig. 2 a A total of $93 \%$ of students felt that the radiologist was critically or very important. b A total of $98 \%$ of students felt that a basic understanding of radiology concepts was important to their future medical practice

When asked how confident they will likely feel in their ability to interpret basic radiographs at the end of their medical school training, $51 \%$ of students responded "slightly confident"(35\% of pre-clerkship, $40 \%$ of clerkship) or "not at all confident" ( $12 \%$ of pre-clerkship, $15 \%$ of clerkship). Only $7 \%$ of students responded "completely confident" or "very confident". Over $91 \%$ of students responded that "a little more teaching is needed" ( $47 \%$ of pre-clerkship, $45 \%$ of clerkship) or "much more teaching is needed" ( $40 \%$ of preclerkship, $50 \%$ of clerkship).

Didactic lectures were the preferred medium of instruction for $26 \%$ of students (37\% of pre-clerkship, $13 \%$ of clerkship), group learning sessions for $28 \%$ (19\% of pre-clerkship, $36 \%$ of clerkship), web-based learning modules for $34 \%$ (25\% of pre-clerkship, $43 \%$ of clerkship), tutorial-based cases for $8 \%$ (12\% of pre-clerkship, $6 \%$ of clerkship), and another method for $4 \%$ ( $7 \%$ of pre-clerkship, $2 \%$ of clerkship) (Fig. 3).

Forty-five percent of students preferred didactic lectures for the delivery of radiology teaching. Thirty-two percent requested group learning sessions, $13 \%$ web-based tutorials, $8 \%$ case-based learning, and $2 \%$ preferred another method.

\section{Discussion}

Clinical radiology is a multidisciplinary field that is inextricably linked to gross anatomy, pathology, and clinical medicine as a whole. The need for imaging be justified based on an appropriate history and physical exam, and the teaching of radiology should be considered an essential aspect of education in the other morphological sciences.

The data confirmed that the majority of medical students view radiology as an important component of healthcare. Whether this is intuitive or is being taught is uncertain, but despite a low number of explicit radiology lectures in our curriculum, many other lecturers will briefly mention the role of the radiology in the workup of a patient. Perhaps as a result, we found that the vast majority of students believe that a basic understanding of general radiology concepts and appropriateness criteria is very important to their future medical practice. We believe every medical graduate should be able to identify the type of study they are attempting to interpret. In the case of plain radiographs, we also believe it is reasonable to expect any graduate to be able to describe how they

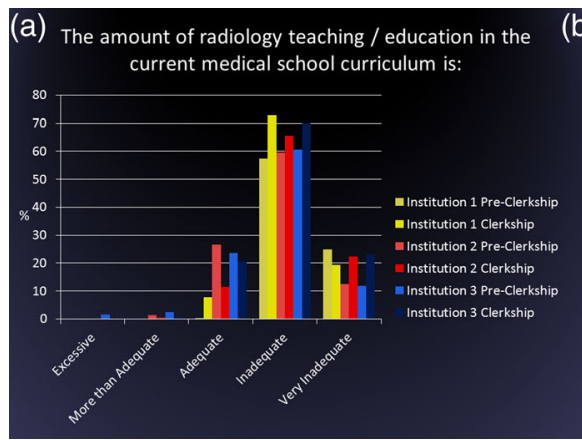

(b) At the end of your medical school training, how confident will you feel in your ability to adequately interpret the findings on basic radiology studies, such as chest $x$-rays, abdominal $x$-rays, and bone $x$ radiology studies, such as chest $\mathrm{x}$-rays,
rays?

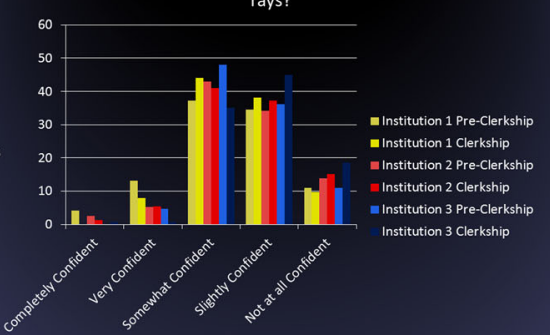

(C) In terms of the amount of radiology education / teaching in the curriculum, which of the following statements do you agree with?

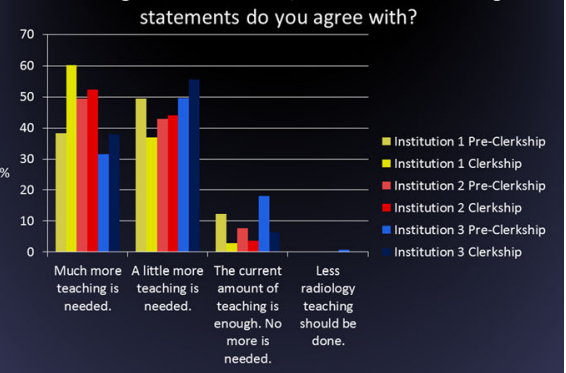

Fig. 3 a A total of $83 \%$ felt that the amount of radiology education in the medical school curriculum is either inadequate or very inadequate. $\mathbf{b}$ When asked how confident they will feel in their ability to interpret basic radiographs at the end of their medical school training, $51 \%$ of students responded slightly confident or not at all confident. Confidence appeared to decrease with student seniority. c A total of $91 \%$ of all students asked felt that much or a little more teaching was needed 
are acquired and name standard projections. Moreover, an awareness of standard radiographic densities (i.e., air, fluid, soft tissue, bone, and metal) and the appearance of some normal anatomic structures was expected within the spectrum of "basic" abilities described to our students. Lastly, an awareness of the appearance of common pathologies and what study should be ordered if they are suspected is important.

As a direct reflection of the value placed on the understanding of radiology, medical students felt that the amount of its teaching in the curriculum was inadequate and that more teaching is needed. They feel only "somewhat" or "slightly confident" in their ability to interpret basic $\mathrm{x}$-rays by the end of medical school. Interestingly, their confidence to interpret $\mathrm{x}$-rays decreases as they progress in their education. We believe that this may be attributable to the realization that there is less radiology education in the curriculum than was initially anticipated and it is not until their clerkship years that they are pressed to interpret $\mathrm{x}$-rays in a clinical environment. In other words, clerkship students have acquired a better sense of what they do not know well, whereas that deficit had not been challenged previously. It may be unrealistic to expect students to have complete confidence with any number of preclinical seminars, but we do believe that arming students with introductory knowledge would motivate self-directed supplementation and also set expectations for their competency. The importance of imaging becomes clearer to a trainee as his/her clinical acumen grows and thus the effectiveness of facilitated learning does as well. At the same time, senior students with superior acumen are also likely more aware of their deficiencies in radiological competency. The confidence that should proceed from comfort with radiology is lacking due to insufficient preparedness, which they recognize.

At some of our institutions, student-run radiology interest groups organized introductory radiology seminars consisting of basic radiology lectures. These were ideally given by radiology residents or fourth-year medical student and staff radiologists when availability was limited. An average of five didactic seminars is given per year, and these occur after hours due to the unavailability of lecture time. As many as $80 \%$ of medical students attend on a voluntary basis, which usually represents the largest attendance of any student group event. Though these sessions are didactic, interaction and participation are encouraged. The ability to hold these seminars in person, we believe, improves the standing of the radiology department within the greater medical school and increases exposure to radiology among medical students. It is important for the future of radiology to ensure that radiologists and radiology residents are visible and relevant to clinical specialties, and one method is to increase awareness of radiology among medical students. Feedback cards and/ or written communications to the interest groups following each lecture were all very positive, as was ad hoc verbal feedback.

Designed to parallel the pre-clerkship curriculum, the seminars produced are introduction to radiology as a specialty, introduction to neuroimaging, introduction to chest imaging, introduction to abdominal imaging, and introduction to MSK imaging. Our hope is to incorporate the lectures into the medical school curriculum. Moreover, radiology residents will continue to give these lectures as it represents an excellent opportunity for our trainees to experience the role of a medical educator.

We feel this is the bare minimum of education in image interpretation and test appropriateness, a skill of importance in the daily life of a hospital-based resident and physician. As this is a new initiative, it remains to be seen whether these seminars will have an impact on student confidence in study interpretation and their perceptions of radiology as a specialty. However, medical students at all levels certainly feel there is a need, and this feeling of need appears to intensify as students enter the hospital for clerkship. Our current goal is to improve the amount and quality of radiology education in the curriculum by working closely with medical students and using their feedback to help mold the delivery of education.

In addition to the resident-run seminars described, our radiology departments all provide instruction in bedside ultrasound and radiologic anatomy laboratories, though there is typically only one of each session. To our surprise, the majority respondents indicated they would prefer to receive additional teaching in a didactic lecture format. We had anticipated that the modern student would prefer a web-based or group learning structure. We received ad hoc feedback at the end of our seminars on feedback cards as well as positive feedback from mandatory online evaluations on radiology lectures and integrated anatomy labs. Students specifically commented on the fact that they felt considerably more confident in the interpretation of plain radiography and unenhanced computed tomography.

As postgraduate medical programs strive to keep attracting the strongest applicants to train in radiology, we feel that this assessment is particularly timely. Briefly, as US programs move toward implementing the DIRECT pathway, exposure to radiology in medical school is important in order to make students aware of not only radiology in general but also interventional radiology specifically. A survey of awareness and teaching regarding the subspecialty may bolster the importance of instituting similar programs at other centers or placing emphasis on interventional radiology teaching. We note that the program with the least instruction in radiology (institution 1) 
produced the most responses demanding more radiology education, indicating that the current standard was inadequate and expressing that their confidence was lowest. Conversely, the program with the most teaching (institution 3) showed the opposite. Importantly, demand was still high with the current standard at any of these institutions.

Given the ease with which surveys can be constructed and distributed after appropriate research ethics considerations, repeat assessment is feasible. Moreover, a survey can be applied to any undergraduate medical school to assess the status of radiology education. Importantly, this benefit should be gauged objectively, with assessments and feedback on progression and deficits in adequacy of students' understanding of basic radiology concept and appropriateness criteria. Should there be demonstrable benefit from this didactic instruction, our hope is that curriculum designers with an interest in undergraduate medical education, as well as academic radiology departments, will seize the opportunity to impact the next generation of physicians.
Acknowledgments There was no financial support for this study.

\section{References}

1. Gunderman RB, Siddiqui AR, Heitkamp DE, Kipfer HD. The vital role of radiology in the medical school curriculum. Am J Roentgenol. 2003;180(5):1239-42.

2. Holt NF. Medical students need more radiology education. Acad Med. 2001;76(1):1.

3. Jeffrey DR, Goddard PR, Callaway MP, Greenwood R. Chest radiograph interpretation by medical students. Clin Radiol. 2003;58(6): 478-81.

4. Butter J, Grant TH, Egan M, Kaye M, Wayne DB, Carrion-Carire V, et al. Does ultrasound training boost year 1 medical student competence and confidence when learning abdominal examination? Med Educ. 2007;41(9):843-8.

5. Scheiner JD, Novelline RA. Radiology clerkships are necessary for teaching medical students appropriate imaging work-ups. Acad Radiol. 2000;7(1):40-5.

6. Dienstag JL. Evolution of the new pathway curriculum at Harvard medical school the new integrated curriculum. Perspect Biol Med. 2011;54(1):36-54.

7. Sugand K, Abrahams P, Khurana A. The anatomy of anatomy: a review for its modernization. Anat Sci Educ. 2010;3(2):83-93. 